\title{
Association of DNA-PK activity and radiation-induced NBS1 foci formation in lymphocytes with clinical malignancy in breast cancer patients
}

\author{
MASANORI SOMEYA ${ }^{1}$, KOH-ICHI SAKATA ${ }^{1}$, YOSHIHISA MATSUMOTO ${ }^{2}$, \\ HIROSHI TAUCHI $^{3}$, HIDEAKI NARIMATSU ${ }^{4}$ and MASATO HAREYAMA ${ }^{1}$ \\ ${ }^{1}$ Department of Radiology, Sapporo Medical University, School of Medicine, Sapporo; ${ }^{2}$ Research Laboratory for \\ Nuclear Reactors, Tokyo Institute of Technology, Tokyo; ${ }^{3}$ Department of Environmental Sciences, \\ Faculty of Science, Ibaraki University, Mito; ${ }^{4}$ Sapporo Rinsho, Sapporo, Japan
}

Received May 9, 2007; Accepted July 13, 2007

\begin{abstract}
DNA double-strand break (DSB) is one of the most deleterious lesions induced by DNA damaging agents. DSB repair pathway is implicated in maintaining genomic integrity via suppression of genetic instability and neoplastic transformation. DNA-dependent protein kinase (DNA-PK) has a pivotal role in DNA DSB repair. The Nijmegen breakage syndrome protein (NBS1), essential for DSB repair, re-localizes into subnuclear structures upon induction of DNA damage by ionizing radiation, forming so-called ionizing radiation-induced foci (IRIF), which is visualized by immunostaining. We measured DNA-PK activity and the number of persistent NBS1 IRIF per nucleus $24 \mathrm{~h}$ after irradiation of peripheral blood lymphocytes (PBL) from patients with sporadic breast cancer. Chromosomal aberrations were examined by cytogenetic methods. We examined the relationship between these measurements and clinical characteristics of patients such as tumor size, lymph node metastasis and nuclear grade of cancer cells. A higher number of NBS1 IRIF or lower DNA-PK activity correlated with higher chromosome instability. Patients whose PBL had lower DNA-PK or higher NBS1 IRIF had aggressive cancer phenotypes such as a larger tumor, higher nuclear grade and positive axillary lymph node metastasis. The combination of DNA-PK activity and NBS1 IRIF were useful for predicting lymph node metastasis. The ability of DSB repair in PBL is related to aggressive breast cancer phenotypes. Axillary lymph node dissection can be avoided by examining DNA-PK
\end{abstract}

Correspondence to: Dr Koh-ichi Sakata, Department of Radiology, Sapporo Medical University, School of Medicine, Sapporo, Japan E-mail: sakatako@sapmed.ac.jp

Key words: breast cancer, Nijmegen breakage syndrome protein, DNA-dependent protein kinase, axillary lymph node metastasis, peripheral blood lymphocytes activity and NBS1 IRIF of PBL, which can contribute to improving the quality of life of breast cancer patients.

\section{Introduction}

One of the hallmarks of malignant transformation is genomic instability, which promotes a wide range of mutations, such as chromosome deletions, gene amplifications, translocations and polyploidy. The presence of genomic instability in cells is known to play an important role in multistage carcinogenesis (1) and genes involved in the maintenance of genomic stability can be considered as cancer suppressor genes. Repair of various types of DNA damage is critical for genomic instability. Of these, DNA double-strand break (DSB) is believed to be one of the most serious damage induced by DNA damaging agents (2).

In DNA DSB repair, non-homologous end-joining (NHEJ) is one major mechanism (3). In the NHEJ pathway, DSBs are directly, or after processing of the DNA ends, rejoined at an appropriate chromosomal end and DNA-dependent protein kinase (DNA-PK) plays an important role in DNA DSB repair by NHEJ throughout the cell cycle (4). DNA-PK is a serine/threonine kinase, which is composed of DNA-PK catalytic subunit (DNA-PKcs) and heterodimer of Ku70 and Ku86. DNA-PK binds DSBs in DNA, phosphorylates and activates a DNA-binding protein, such as XRCC4 and DNA ligase IV, p53 and several transcription factors. Subsequently, Ligase IV repairs DNA DSB (5).

The Nijmegen breakage syndrome protein (NBS1), is a component of a protein complex containing Mre11 and Rad50. Hypomorphic mutations in $\mathrm{Nbsl}$ are known to lead to Nijmegen breakage syndrome in humans, known as a radiation hypersensitive disease (6). This complex plays a role in many processes involved in maintaining genomic stability (7). Though its precise function is still unclear, the NBS1 complex is known to be involved in cell cycle checkpoint activation and repair of DSBs (8). The NBS1 complex plays a pivotal role in preventing genomic instability during DNA replication and DNA DSB repair (6). The NBS1/hMRE11/ 
hRAD50 complex re-localize into subnuclear structures upon the induction of DNA damage by ionizing radiation, the socalled ionizing radiation-induced foci (IRIF) $(9,10)$. These IRIF are known to form at the site of DNA damage $(11,12)$. IRIF can be visualized indirectly through immunostaining with antibodies against the protein of interest, or directly through expression of the protein tagged to a fluorescent protein. The higher fraction of NBS1 IRIF remaining after irradiation may be related to more DNA DSBs unrepaired, indicating a lower ability to repair DNA DSBs. Unrepaired DNA ends might contribute to the development of chromosomal translocations by acting as transposable elements $(13,14)$.

We have previously reported that DNA-PK activity and the number of persistent NBS1 IRIF in peripheral blood lymphocytes (PBLs) is associated with chromosomal instability and the risk of sporadic breast cancer $(15,16)$.

In this study, we measured DNA-PK activity and NBS1 IRIF remaining after irradiation of PBL obtained from patients with sporadic breast cancer. We examined the relationship between these measurements and pathological characteristics of these patients such as tumor size, axillary lymph node metastasis and nuclear grade of cancer cells in invasive breast cancer patients in order to predict clinical malignancy with these measurements.

\section{Patients and methods}

Selection eligibility. All subjects were Japanese. Ninety-six of sporadic breast cancer patients underwent breast conserving surgery and are planning to receive postoperative radiotherapy to conserved breast at Sapporo Medical University. Those who had neither a history of another cancer nor familial breast cancer history were enrolled in this study. The study was approved by the appropriate Committees for Human Rights in Research in our hospital and each patient gave their written informed consent. Exclusion criteria included previous or current use of chemotherapy, radiation therapy, or immunosuppressive medications.

Pathological evaluation of the patients. All breast cancer patients who had breast conserving surgery and axillary lymph node resection and pathological diagnosis of invasive ductal cancer were confirmed. Size of the tumor, expression of hormone receptors and lymph node metastasis were also examined. Nuclear grade (such as histological grade of malignancy) in breast cancer was evaluated by combining nuclear atypia and mitotic counts according to the Japanese breast cancer classification (17). Nuclear grade was divided into 3 groups; grade 1 for low-risk, 2 for intermediate risk and 3 for high risk malignancy, respectively.

Blood collection and PBL separation. Peripheral blood (20 ml) was collected with a sterile heparinized tube from each individual before radiotherapy began. Peripheral blood lymphocytes (PBLs) were separated with lymphoprep (Nycomed Pharma AS), centrifuged at $1500 \mathrm{rpm}$ (300 x g) for $30 \mathrm{~min}$ at $4^{\circ} \mathrm{C}$, washed twice with phosphate-buffer saline.

PBL lysis, protein extraction, DNA-PK assay. Protein extraction and DNA-PK assay was done as described in our previous report (16). PBL was thawed with high salt buffer and the suspension was lysed by three rounds of freeze-thaw cycle and clarified by centrifugation at $15,000 \mathrm{rpm}(18,000 \mathrm{x} \mathrm{g})$ for $7 \mathrm{~min}$ at $4^{\circ} \mathrm{C}$. Protein concentration was assayed using a BCA protein assay kit (Pierce) with bovine serum albumin as the standard. The PBL cell lysates were diluted to $0.25 \mathrm{mg} / \mathrm{ml}$ with high salt buffer. The lysate was mixed with kinase assay buffer, synthetic peptide hp53-S15 (sequence: EPPLSQEAF ADLWKK; synthesized in Sawady Biotechnology) and with or without sonicated salmon sperm DNA. This reaction mixture was incubated at $37^{\circ} \mathrm{C}$ for $10 \mathrm{~min}$. The reaction was stopped by the addition of $30 \%$ acetic acid and absorbed onto a phosphocellulose filter disc $(2.3 \mathrm{~cm}$ in diameter, Whatman). The filter discs were washed in $15 \%$ acetic acid and in $99 \%$ ethanol and the remaining radioactivity was counted in a liquid scintillation counter. The net phosphorylation of hp53-S15 was calculated as phosphate incorporation in reaction with DNA minus that in reaction without DNA, divided by the specific radioactivity of ATP.

Immunofluorescent staining for radiation-induced NBS1 foci in $P B L s$. Ionizing radiation-induced NBS1 foci formation in PBLs were also measured in 46 out of 96 patients. Separated PBLs were incubated with RPMI-1640 medium (Sigma Aldrich), supplemented with $20 \%$ fetal calf serum. 4 Gy were irradiated with $120 \mathrm{kV}$ X-ray at $1.17 \mathrm{~Gy} / \mathrm{min}$. PBLs were applied on slide glass with centrifugation in 1, 4 and $24 \mathrm{~h}$ after irradiation, respectively. PBLs diluted to appropriate numbers were grown on a glass slide and fixed with cold methanol for $20 \mathrm{~min}$, rinsed with cold acetone for $10 \mathrm{sec}$ and then air-dried. Anti-NBS1 (Novus Biologicals) were used as the primary antibody. Alexa488-conjugated anti-rabbit IgG (Molecular Probes) were used for visualization of foci with anti-NBS1 antibody. Slides were mounted with antifade reagent (Mounting medium, Dako).

NBS1 foci were observed with an Olympus fluorescent microscope under 10x100 oil immersion. For quantification of foci, clear and easily distinguished dots of certain brightness were counted as positive foci. The number of NBS1 foci was counted in 200 cells of the sample at each time point by visual inspection and average number of foci per cell was calculated. A representive example and time course were indicated in Fig. 1A and B. Since NBS1 foci were gradually larger and more distinguishable, here we used average number of NBS1 foci at $24 \mathrm{~h}$ after irradiation for NBS1 foci quantification.

Chromosomal aberrations in PBLs. Spontaneous chromosomal aberration in PBLs were observed by Giemsa staining in 30 patients. The procedure was described previously (15). Two hundred metaphase cells from each individual were analyzed and the numbers of dicentric chromosomes and chromosome breaks were counted. Chromosome breaks not accompanying a dicentric chromosome were recorded as excess fragments.

Statistical methods. The unpaired t-test was used to compare DNA-PK activity between groups. All statistical tests were two-sided. Multiple regression analysis was used to clarify significant variables which correlate with lymph node metastasis. All statistical computing was done with StatView version 4.58 (Abacus Concepts). 
A

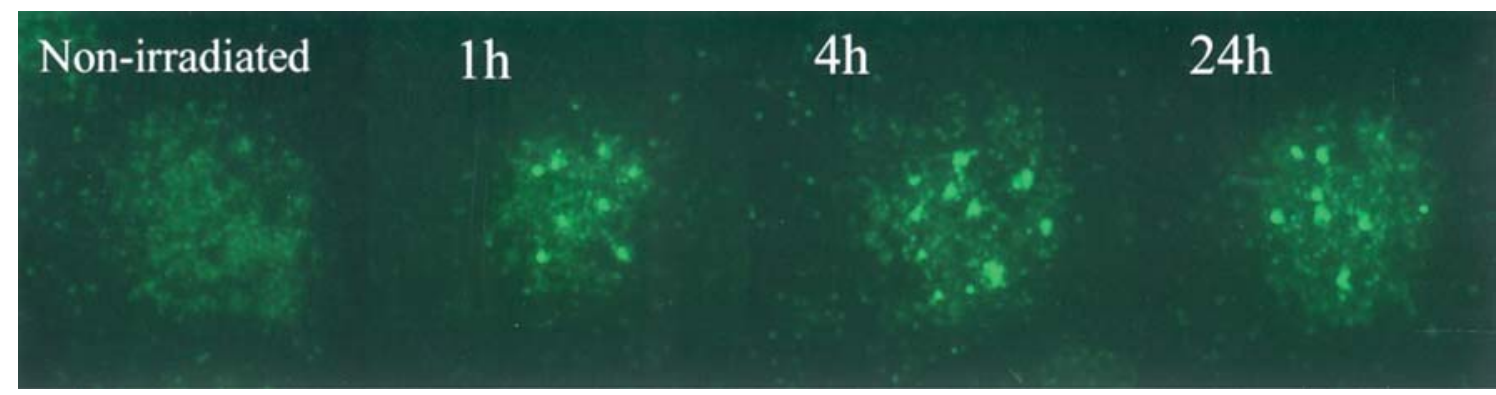

B

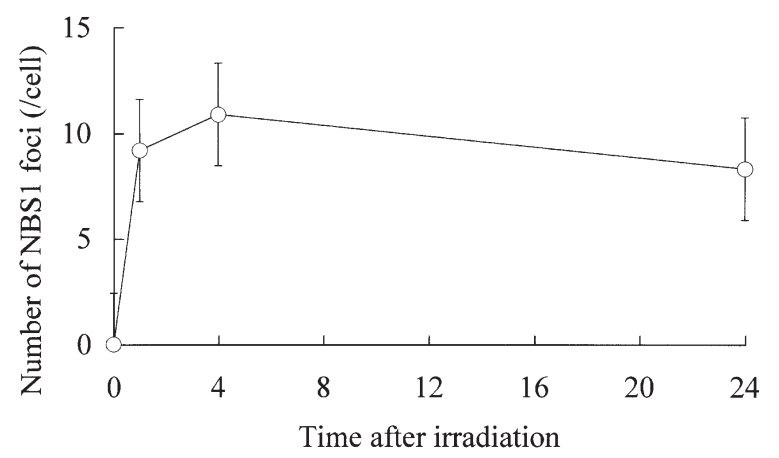

C

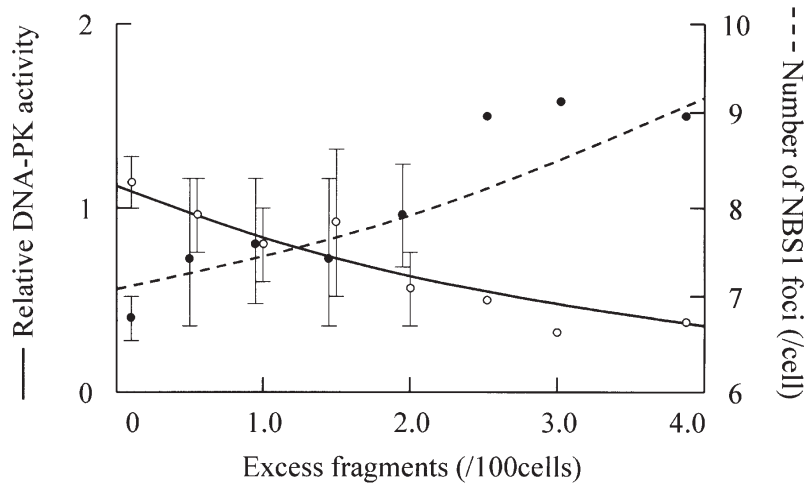

Figure 1. (A) Representive example of formation of radiation-induced NBS1 foci in PBLs. PBLs were unirradiated or irradiated with 4 Gy and fixed at 1,4 and $24 \mathrm{~h}$ after irradiation. Immunofluorescence staining was then performed with NBS1 antiserum. (B) Kinetics of NBS1 foci at time course. Bars were indicated as standard deviation. (C) Association of chromosome aberration and DNA-PK activity ( $\bigcirc$ ) and NBS1 foci (l). The DNA-PK activity is expressed as a ratio of activity of each sample to that of the internal control. Lower DNA-PK activity and higher NBS1 foci were associated with higher chromosome breaks. Lines indicate a regression curve.

\section{Results}

DNA-PK activity in PBL. The DNA-PK activity of PBL in patients with invasive breast cancer was $8.7 \pm 4.5 \mathrm{pmol}$.

Kinetics of NBS1 IRIF formation in PBL. Fig. 1A shows representative photographs of NBS1 IRIF of PBLs. There was no obvious foci detected for unirradiated control of any subject. As an internal control, PBLs from the same individual were simultaneously studied in the first 20 experiments and the number of NBS1 IRIF of internal control had a good correlation in each experiment. The formation of NBS1 IRIF was examined at 1, 4 and $24 \mathrm{~h}$ after 4 Gy of X-ray irradiation (Fig. 1B). The number of NBS1 IRIF increased at 1 to $4 \mathrm{~h}$ after irradiation, then gradually decreased until $24 \mathrm{~h}$ after irradiation. The number of cells with IRIF correlated well with the average number of foci per cell (data not shown). So, we only calculated the average number of foci per cell.

The association of spontaneous chromosome aberration with DNA-PK activity and NBS1 foci. Fig. 1C showed a relationship between spontaneous chromosomal aberration and DNA-PK activity and NBS1 foci. Lower DNA-PK activity and a higher retention of NBS1 foci were associated with a higher frequency of excess fragments $(r=-0.548$ and $r=0.488$ for DNA-PK activity and NBS1 foci, respectively).

Relationship between nuclear grade and DNA-PK activity in $P B L$. The reproducibility and reliability of DNA-PK assay has been described in our earlier publication (15). The sample of the same person was simultaneously run for DNA-PK assays as an internal control and relative DNA-PK activities were calculated for each assay.

By combining the nuclear atypia and mitotic counts, nuclear grades were defined as the sum of scores for the nuclear atypia (1 for low-degree atypia; 2 for intermediatedegree atypia; 3 for high-degree atypia) with the scores for the mitotic counts per 10 high-power fields (x40 objective lens) ( 1 for $0-4$ mitoses; 2 for $5-9$ mitoses; 3 for $>10$ mitoses). The nuclear grade was 1,2 and 3 when the sum of scores for the nuclear atypia and those for mitotic counts were 2-3, 4 and 5-6, respectively (17).

Fig. 2A shows the comparison of DNA-PK activity of PBL between patients whose tumor cells had nuclear grade 1 and those whose tumor cells had nuclear grade $2+3$. Average of relative DNA-PK activity was $0.67 \pm 0.28$ in nuclear grade 1 and $0.55 \pm 0.21$ in nuclear grade $2+3$. Patients with nuclear grade $2+3$ disease had marginally lower DNA-PK activity than those with nuclear grade $1(\mathrm{P}=0.082)$.

Association between DNA-PK activity and breast cancer stage. Fig. 2B shows the comparison of DNA-PK activity of PBL between patients with T1 tumor and those with T2 tumor. Average of relative DNA-PK activity was $0.67 \pm 0.28$ in $\mathrm{T} 1$ and $0.54 \pm 0.12$ in T2. Patients with $\mathrm{T} 1$ disease had marginally lower DNA-PK activity than those with T2 disease $(\mathrm{P}=0.093)$.

Association between DNA-PK activity in PBL and axillary lymph node metastasis. Fig. 2C demonstrates the comparison 
A

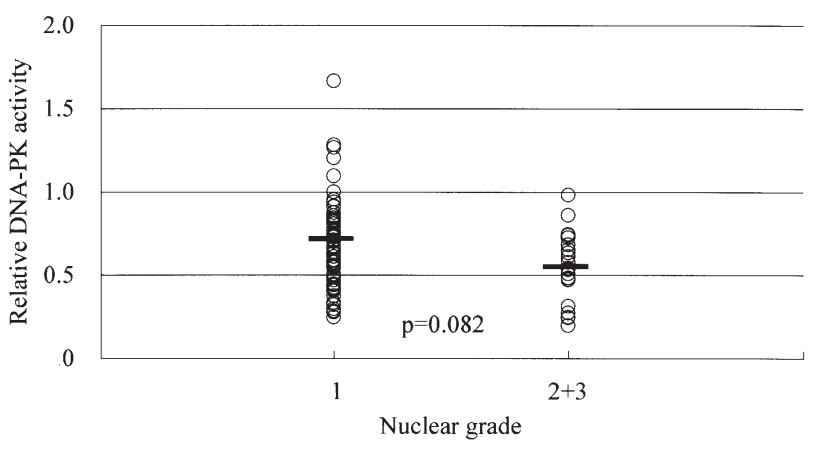

B

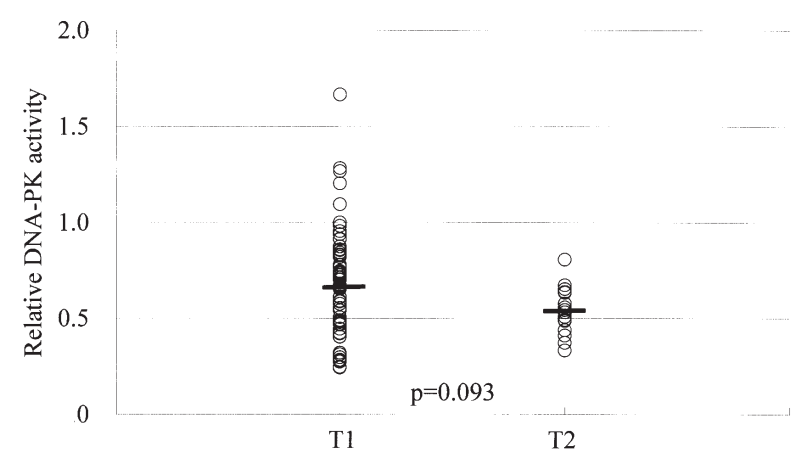

C

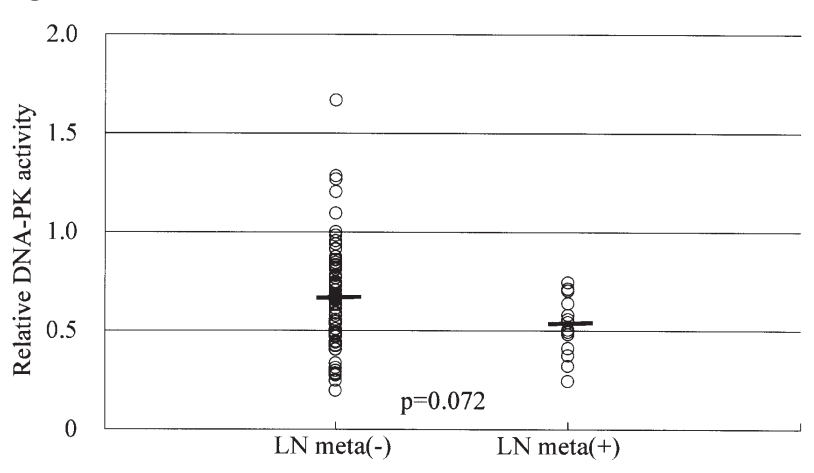

Figure 2. The relationship between clinical characteristics and DNA-PK activity of PBL in patients with sporadic breast cancer. The DNA-PK activity is expressed as a ratio of activity of each sample to that of the internal control. The horizontal bar indicates the mean value. (A) DNA-PK activity in the PBLs according to nuclear grade in a breast cancer specimen $(\mathrm{P}=0.082)$. (B) DNA-PK activity in the PBLs according to T stage $(\mathrm{P}=0.093)$. (C) DNA-PK activity in the PBLs according to the existence of axillary lymph node metastasis $(\mathrm{P}=0.072)$.

of DNA-PK activity of PBL between patients with axillary lymph node metastasis and those without axillary lymph node metastasis. Average of relative DNA-PK activity was $0.52 \pm 0.15$ in patients with axillary lymph node metastasis and $0.66 \pm 0.28$ in patients without axillary lymph node metastasis. Patients with axillary lymph node metastasis had marginally lower DNA-PK activity than those without nuclear axillary lymph node metastasis $(\mathrm{P}=0.072)$.

Association between NBS1 foci formation in PBL and axillary lymph node metastasis. Fig. 3 demonstrates the comparison of NBS1 IRIF of PBL at $24 \mathrm{~h}$ after irradiation between patients with axillary lymph node metastasis and those without

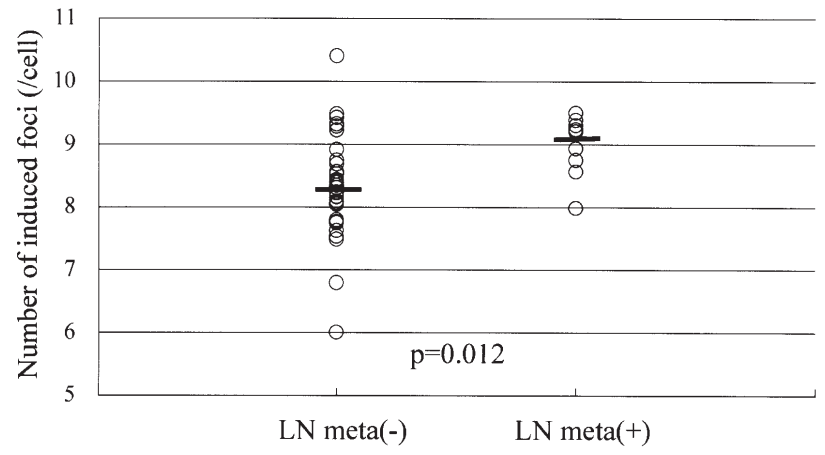

Figure 3. The relationship between axillary lymph node metastasis and radiation-induced NBS1 foci in PBLs at $24 \mathrm{~h}$ after irradiation with 4 Gy $(\mathrm{P}=0.012)$. The horizontal bar indicates the mean value.

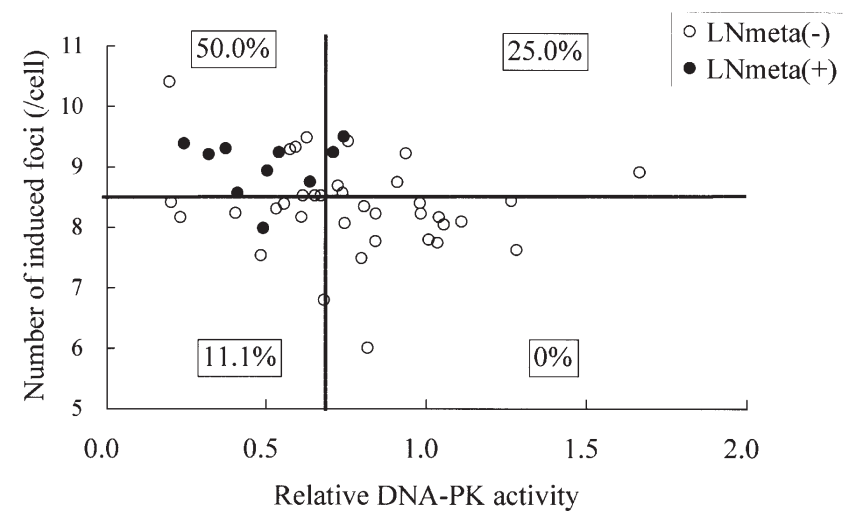

Figure 4. The association of lymph node metastasis, DNA-PK activity and radiation-induced NBS1 foci. The DNA-PK activity is expressed as a ratio of activity of each sample to that of the internal control.

axillary lymph node metastasis. Average number of NBS1 IRIF at $24 \mathrm{~h}$ after irradiation was $9.01 \pm 0.46$ in patients with axillary lymph node metastasis and $8.33 \pm 0.78$ in patients without axillary lymph node metastasis. Patients with axillary lymph node metastasis had a significantly higher NBS1 IRIF than those without nuclear axillary lymph node metastasis $(\mathrm{P}=0.012)$.

Prediction of lymph node metastasis by combination of DNA$P K$ and NBS1 IRIF. We divided the patients into 2 groups based on the median value of DNA-PK activities and number of NBS1 foci. In the group that had lower DNA-PK activity and higher NBS1, half of the patients (7 out of 14) had lymph node metastasis, whereas no patients had lymph node metastasis in the higher DNA-PK activity and lower NBS1 IRIF group (Fig. 4). Multiple regression analysis shows that lower DNA-PK activity $(\mathrm{P}=0.009)$ and the lower number of radiation-induced NBS1 foci $(\mathrm{P}=0.001)$ are significant factors that predict lymph node metastasis and that other factors (age, ER status, T stage and nuclear grade) did not correlate with lymph node metastasis (Table I).

\section{Discussion}

We previously demonstrated that DNA-PK activity in PBLs of patients with invasive breast cancer or uterine cervix cancer 
Table I. Variables that predict axillary lymph node metastasis by multiple regression analysis.

\begin{tabular}{|c|c|c|c|c|c|}
\hline \multirow[b]{2}{*}{ Variable } & \multirow[b]{2}{*}{$\mathrm{CC}$} & \multirow[b]{2}{*}{ SE } & \multirow[b]{2}{*}{$\mathrm{P}$} & \multicolumn{2}{|c|}{$95 \% \mathrm{CI}$} \\
\hline & & & & $\begin{array}{l}\text { Lower } \\
\text { bound }\end{array}$ & $\begin{array}{l}\text { Upper } \\
\text { bound }\end{array}$ \\
\hline Age & 0.002 & 0.161 & 0.978 & -0.018 & 0.019 \\
\hline Estrogen receptor & -0.667 & 0.356 & 0.078 & -1.418 & 0.084 \\
\hline T stage & 0.180 & 0.165 & 0.29 & -0.168 & 0.529 \\
\hline Nuclear grade & -0.229 & 0.159 & 0.167 & -0.565 & 0.106 \\
\hline $\begin{array}{l}\text { DNA-PK activity } \\
\text { lower/higher group }\end{array}$ & -0.476 & 0.161 & 0.009 & -0.476 & -2.947 \\
\hline $\begin{array}{l}\text { NBS1 foci } \\
\text { lower/higher group }\end{array}$ & 0.575 & 0.144 & 0.001 & 0.272 & 0.879 \\
\hline
\end{tabular}

CC, correlation coefficient; SE, standard error; $C I$, confidence interval; $\mathrm{n}=46 ; \mathrm{R}^{2}=0.691 ; \mathrm{P}=0.021$.

was significantly higher than in normal healthy volunteers (15). We also showed that the number of persistent radiationinduced NBS1 foci in PBLs of patients with invasive breast cancer was significantly higher than in normal healthy volunteers (16). Age and smoking had no association with DNA-PK activity or NBS1 foci in PBLs. These results indicate that the number of persistent radiation-induced NBS1 foci and DNA-PK activity in PBL are associated with the risk of breast cancer. In this study, we examined whether there may be a possible link between clinical and pathological characteristics of breast cancer and DNA DSB repair capability measured with NBS1 IRIF and DNA-PK activity.

Results of Fig. 1A and B establish the influence of time after induction of DNA damage by irradiation on NBS1 IRIF. A time-dependent increase in IRIF is seen in all PBLs investigated. In general, the number of NBS1 IRIF peaked at $4 \mathrm{~h}$ and decreased to $24 \mathrm{~h}$. The number of IRIF per nucleus varies among patients, indicating that patients had a different ability of DNA DSBs repair since the fraction of NBS1 IRIF remaining after irradiation may be related to DNA DSBs unrepaired (16). The DNA-PK activity of PBL in patients with invasive breast cancer also varied considerably (Fig. 2) (15).

Then, we investigated the relationship among NBS1 IRIF, DNA-PK activity and chromosomal aberrations by cytogenetic methods. We showed an association among NBS1 IRIF, DNA-PK activity and spontaneous yield of excess fragments. A higher number of NBS1 IRIF or lower DNA-PK activity correlated with higher chromosome instability (Fig. 1C). The higher fraction of NBS1 IRIF remaining after irradiation may be related to more DNA DSBs unrepaired, indicating a lower ability to repair DNA DSBs (16). Reduced DNA-PK activity can profoundly affect the ability to repair double-strand breaks, resulting in the perpetuation of chromosome damage. Unresolved signal ends might contribute to the development of translocations by acting as transposable elements $(13,14)$. Our results indicate that the lower DNA-PK activity or the higher fraction of NBS1 IRIF remaining after irradiation is related with chromosomal instability. Repair of various types of DNA damage is critical for genomic instability. Of these, DNA double-strand break (DSB) is believed to be one of the most serious damage induced by DNA damaging agents (2). Genes involved in DNA DSB repair plays an important role in the maintenance of genomic stability (18).

Fig. 2A demonstrates that patients with a nuclear grade of $2+3$ had lower DNA-PK activity than patients with a nuclear grade of 1. Fig. 2B demonstrates that patients with T2 tumor had lower DNA-PK activity than patients with T1. Patients with axillary lymph node metastasis had lower DNA-PK activity (Fig. 2C) or significantly higher NBS1 IRIF (Fig. 3) than those without axillary lymph node metastasis. In this way, our results demonstrated that patients with the lower DNA-PK or higher NBS1 IRIF had aggressive cancer phenotypes such as a larger tumor, a higher nuclear grade, and positive axillary lymph node metastasis. Aggressive cancer phenotypes are a manifestation of many different genetic alterations that promote rapid proliferation and metastasis (19). Genetic instability prompted the loss or activation of a number of critical genes, such as those involved in cell proliferation, differentiation and apoptosis (20-23). Goode et al demonstrated that polymorphisms of Ligase IV significantly influenced survival among women with breast cancer participating in a British population-based study (24). Ligase IV plays an important role in non-homologous end-joining (NHEJ) which is one major mechanism of DNA DSB repair.

From a standpoint of clinical application, close attention was paid to the relationship among DNA-PK activity, NBS1 IRIF and axillary lymph node metastasis. Major changes have recently occurred in the rationale for and approach to the management of breast cancer. Breast-conserving operation and postoperative radiotherapy have largely replaced total mastectomy as the standard treatment of choice for many primary breast cancers, contributing to the improvement of quality of life of patients (25). In operations of breast cancer, axillary lymph node dissection has also been performed for axillary staging providing valuable information to the planning of adjuvant therapy as well as an excellent regional disease control (26). Patients with positive axillary nodal disease have a worse prognosis than those with negative nodal disease. So, patients with positive axillary nodal disease receive systemic adjuvant therapy (27). However, axillary lymph node dissection is associated with significant arm morbidity, chronic lymphoedema being the most 
notorious form (26). We demonstrated that the combination of DNA-PK activity and NBS1 foci were useful for the prediction of axillary lymph node metastasis (Fig. 4). In particular, no patients had lymph node metastasis when they had a higher DNA-PK activity and lower NBS1 IRIF. These results indicate that axillary lymph node dissection can be avoided by examining DNA-PK activity and NBS1 IRIF of PBL, which can contribute to improving of quality of life of patients with breast cancer.

In summary, a higher number of NBS1 IRIF or lower DNA-PK activity correlated with higher chromosome instability. Patients whose PBL had lower DNA-PK or higher NBS1 IRIF had aggressive cancer phenotypes such as the higher nuclear grade, a larger tumor and positive axillary lymph node metastasis. This result means that DNA-PK activity and NBS1 foci can detect lymph node metastasis and may be an independent prognostic factor for early stage invasive breast cancer. Axillary lymph node dissection can be avoided by examining DNA-PK activity and NBS1 IRIF of PBL, which can contribute to improving the quality of life of breast cancer patients.

\section{References}

1. Lengauer C, Kinzler KW and Vogelstein B: Genetic instabilities in human cancers. Nature 396: 643-649, 1998.

2. Bryant PE: Enzymatic restriction of mammalian cell DNA: evidence for double-strand breaks as potentially lethal lesions. Int J Radiat Biol 48: 55-60, 1985.

3. Ferguson DO, Sekiguchi JM, Chang S, et al: The nonhomologous end-joining pathway of DNA repair is required for genomic stability and the suppression of translocations. Proc Natl Acad Sci USA 97: 6630-6633, 2000.

4. Lees-Miller SP: The DNA-dependent protein kinase, DNA-PK: 10 years and no ends in sight. Biochem Cell Biol 74: 503-512, 1996.

5. Jeggo PA: Identification of genes involved in repair of DNA double-strand breaks in mammalian cells. Radiat Res 150: S80-S91, 1998.

6. Tauchi H, Matsuura S, Kobayashi J, Sakamoto S and Komatsu K: Nijmegen breakage syndrome gene, NBS1, and molecular links to factors for genome stability. Oncogene 21: 8967-8980, 2002.

7. de Jager $\mathrm{M}$ and Kanaar R: Genome instability and Rad50 (S): subtle yet severe. Genes Dev 16: 2173-2178, 2002.

8. D'Amours D and Jackson SP: The Mre11 complex: at the crossroads of DNA repair and checkpoint signalling. Nat Rev Mol Cell Biol 3: 317-327, 2002.

9. Haaf T, Golub EI, Reddy G, Radding CM and Ward DC: Nuclear foci of mammalian Rad51 recombination protein in somatic cells after DNA damage and its localization in synaptonemal complexes. Proc Natl Acad Sci USA 92: 2298-2302, 1995.

10. Maser RS, Monsen KJ, Nelms BE and Petrini JH: hMre11 and hRad50 nuclear foci are induced during the normal cellular response to DNA double-strand breaks. Mol Cell Biol 17: 6087-6096, 1997.
11. Nelms BE, Maser RS, MacKay JF, Lagally MG and Petrini JH: In situ visualization of DNA double-strand break repair in human fibroblasts. Science 280: 590-592, 1998

12. Tashiro S, Walter J, Shinohara A, Kamada N and Cremer T: Rad51 accumulation at sites of DNA damage and in postreplicative chromatin. J Cell Biol 150: 283-291, 2000.

13. Agrawal A, Eastman QM and Schatz DG: Transposition mediated by RAG 1 and RAG2 and its implications for the evolution of the immune system. Nature 394: 744-751, 1998.

14. Hiom K, Melek M and Gellert M: DNA transposition by the RAG1 and RAG2 proteins: a possible source of oncogenic translocations. Cell 94: 463-470, 1998.

15. Someya M, Sakata K, Matsumoto Y, et al: The association of DNA-dependent protein kinase activity with chromosomal instability and risk of cancer. Carcinogenesis 27: 117-122, 2006.

16. Someya M, Sakata K, Tauchi H, et al: Association of ionizing radiation induced foci of NBS1 with chromosomal instability and breast cancer susceptibility. Radiat Res 166: 575-582, 2006.

17. Tsuda H, Akiyama F, Kurosumi M, Sakamoto G and Watanabe T: Establishment of histological criteria for high-risk node-negative breast carcinoma for a multi-institutional randomized clinical trial of adjuvant therapy. Japan National Surgical Adjuvant Study of Breast Cancer (NSAS-BC) Pathology Section. Jpn J Clin Oncol 28: 486-491, 1998.

18. Burma S, Chen BPC and Chen DJ: Role of non-homologous end joining (NHEJ) in maintaining genomic integrity. DNA Repair 5: 1042-1048, 2006.

19. Mironchik Y, Winnard PT, Jr, Vesuna F, et al: Twist overexpression induces in vivo angiogenesis and correlates with chromosomal instability in breast cancer. Cancer Res 65: 10801-10809, 2005

20. Chin K, de Solorzano CO, Knowles D, et al: In situ analyses of genome instability in breast cancer. Nat Genet 36: 984-988, 2004.

21. Hezel AF, Kimmelman AC, Stanger BZ, Bardeesy N and Depinho RA: Genetics and biology of pancreatic ductal adenocarcinoma. Genes Dev 20: 1218-1249, 2006.

22. Maser RS and DePinho RA: Connecting chromosomes, crisis, and cancer. Science 297: 565-569, 2002.

23. Nishizaki T, DeVries S, Chew $\mathrm{K}$, et al: Genetic alterations in primary breast cancers and their metastases: direct comparison using modified comparative genomic hybridization. Genes Chromosomes Cancer 19: 267-272, 1997.

24. Goode EL, Dunning AM, Kuschel B, et al: Effect of germ-line genetic variation on breast cancer survival in a population-based study. Cancer Res 62: 3052-3057, 2002.

25. Noguchi M: Current controversies concerning sentinel lymph node biopsy for breast cancer. Breast Cancer Res Treat 84: 261-271, 2004.

26. Leidenius M, Leivonen M, Vironen $\mathrm{J}$ and von Smitten K: The consequences of long-time arm morbidity in node-negative breast cancer patients with sentinel node biopsy or axillary clearance. J Surg Oncol 92: 23-31, 2005.

27. Glick JH, Gelber RD, Goldhirsch A and Senn HJ: Adjuvant therapy of primary breast cancer: 4th International Conference on Adjuvant Therapy of Primary Breast Cancer St. Gallen, Switzerland. Ann Oncol 3: 801-807, 1992. 